\title{
Patient outcomes versus financial outcomes: Which should we listen to?
}

The views expressed in this editorial are those of the author and do not necessarily reflect the position of the Canadian Medical Association or its subsidiaries.

utcomes are important in medicine. Traditionally, we relied on our own internal scoring to determine how well we were doing with our care delivery. But we have spent the last two decades making a concerted effort to determine what patientreported outcomes (PROs) can mean for validation of our procedures and protocols. We are still stuck when it comes to interpretation of these scores.

A PRO is the information reported by a patient, but without interpretation of that response by the surrounding health care team. The strength of a PRO is that it pertains directly to the patient's quality of life and functional status and is a patient-based reflection of how well we did with our interventions. Unfortunately a lot of internal patient information may go into an evaluation. Their perceived outcome in a global evaluation after appendectomy may have as much to do with parking and hospital food as it does with the skill of their surgeon. Researchers have accounted for this by using directed questionnaires to interpret patient responses.

The tools or instruments used to measure PROs are patient-reported outcome measures (PROMs) and patientreported experience measures (PREMs). A PROM is a questionnaire administered to determine the patient's health status. A PREM gauges a patient's perception of the health care they have received. These tools give a more objective value to subjective patient input. We use many objective scores for treatment evaluation, including physiologic parameters, markers and radiographs among others, but PROMs are now often being used as adjuncts to these scores.

Hospital administrators have other outcomes in mind. They are more interested in cash flow, and PREMs can be used for economic viability scoring. Some general systems (e.g., EuroQol, 12-Item Short Form Health Survey) measure health-related quality of life that can be used to estimate quality-adjusted life-years (QALYs), which can be used as discussion points for economic evaluation. As surrogate measurements of outcomes over time, QALYs help us to justify expensive interventions or equipment.

Much as described in the article by Rhee and colleagues in this month's issue, ${ }^{1}$ we have increasingly replaced PROs with economic outcomes. It is much easier to examine these data using data-mining techniques and large patient care databases with financial information embedded. This is a double-edged sword. We would like to know if measures are economically viable. In today's cash-strapped hospital environment, it behooves us to promote financially responsible treatment options. Unfortunately, the disconnect between the finance department and patient outcomes will only be accentuated if we do not marry the two in some way. All new technology is going to be more expensive up front. There is no way, for example, that a modern fixation plate with minimal contact points and locking screw technology will be able to compete with a cold milled flat plate on a per cost analysis. New minimally invasive laparoscopy equipment is always more expensive. In the United States, reimbursement is being based on patient satisfaction scores. ${ }^{2}$ But relying only on patient satisfaction scores without considering the overall care picture may be dangerous. Patient satisfaction does not depend on hospital protocols, on compliance with surgical care measures for accreditation or even on whether the procedure is accepted practice.

I fear if we do not get a handle on what to measure and at what time point, and on whether the results are in context, then we will be hard pressed to determine if we actually treat patients adequately.

\section{Edward J. Harvey, MD}

Coeditor, Canadian Fournal of Surgery

Competing interests: E.J. Harvey is the Chief Medical Officer of Greybox Healthcare (Montreal) and Chairman of the Board of NXTSens Inc. (Montreal).

DOI: $10.1503 /$ cjs.006818

\section{References}

1. Rhee C, Lethbridge L, Richardson G, et al. Risk factors for infection, revision, death, blood transfusion and longer hospital stay 3 months and 1 year after primary total hip or knee arthroplasty. Can $\mathcal{f}$ Surg 2018;61:165-76.

2. Lyu H, Wick EC, Housman M, et al. Patient satisfaction as a possible indicator of quality surgical care. FAMA Surg 2013;148:362-7. 